\title{
The Meaning of Space in Sign Language: Reference, Specificity and Structure in Catalan Sign Language Discourse
}

By Gemma Barberà Altimira

Boston/Berlin: De Gruyter Mouton and Ishara Press, 271 pages.

ISBN: 978-1-61451-866-2

Received: 08-07-2018

Accepted: 08-10-2018

Reviewed by Raquel Veiga Busto and Sara Cañas Peña

Universitat Pompeu Fabra

The use of space in the visual modality impacts sign language grammars in multiple ways. The book reviewed here, based on Barberà's doctoral dissertation, is particularly concerned with the effects that the use of signing space has at the semantics-pragmatics interface. Under Barberà's analysis, the signing space is considered a discrete system, as opposed to the real space surrounding the signer. Since referring terms can be directed to different spatial locations that can be picked up later in the discourse, those locations are taken to be abstract points that stand for discourse referents (DR). However, according to the author, with the notable exception of Lillo Martin \& Klima (1990), no prior formalization accounts for the association of spatial locations and meaning at the level of discourse. And that is precisely the first objective pursued by this publication: to fill this gap in the analysis of space in the sign language literature. Additionally, this book seeks to elucidate the specific semantic functions that spatial locations have in Catalan Sign Language (LSC). To meet these goals, Barberà proposes an analysis framed within dynamic semantics, drawing on Discourse Representation Theory (DRT) (Kamp and Reyle 1993) as the main tool to model the semantics of LSC discourse. Empirically, the data analyzed comes from a small-scale LSC corpus, but information about other sign languages (SLs) is also discussed throughout the book.

Chapter 1 is devoted to present the main objectives of the research, and to describe the methodology that has been used. After providing an overview of sign language research, Barberà highlights the scarcity of studies examining the formal semantics-pragmatics interface in SLs, which is precisely the domain in which this book is situated.

Chapter 2 introduces the reader to the two main conceptions of space found in the sign language literature, namely: the so-called spatial mapping view (Liddell 1990, 2003) and the r-loci perspective (Lillo-Martin \& Klima 1990). The 
former account considers spatial locations as mental projections of the referents in space and, therefore, they are taken to be part of a gestural continuum that cannot be phonologically specified. Under this view, no difference is made between actual locations (the real world) and the discourse. For the r-loci view, on the other hand, spatial locations have a grammatical status: they are considered to be the overt morphological expression of referential indices (variables whose content emanates from discourse). Importantly, from this perspective, reference is made exclusively to discourse, that is, to the signing space, which is considered to be a linguistic construct that differs from the real space around the signer. The author explicitly supports the r-loci view, providing further evidence for this account in all the subsequent chapters.

Chapter 3 is dedicated to the use of space in non-descriptive locations. Non-descriptive locations, unlike descriptive ones, do not make a free use of the signing space and thus they are localized arbitrarily to identify the arguments of the verb. In this chapter, the author describes the three spatial planes - horizontal, frontal and midsagittal — and the set of linguistic mechanisms — manual and nonmanual- used in LSC to establish spatial locations. Those signs directed to different parts of spatial planes contribute to the establishment of (p). (p) is a grammatical morpheme that consists in an abstract point in space and functions as a clitic pronoun. The direction where the physical point is established is completely irrelevant for the LSC grammar. Yet, there are two directions (upper and lower), concerning the frontal plane, which are grammatically relevant. [low] is the default feature signs are associated with; however, the feature [up] is the marked location for denoting concrete meanings, such as locatives, hierarchical relations, non-presence in the immediate physical context and non-specificity.

The function of the spatial morpheme (p) is further examined in Chapter 4. In line with r-loci view of spatial locations, (p) is taken to be the overt manifestation of discourse referents (DRs). Therefore, in LSC, identity relations between variables in the discourse model are achieved through their coincidence with the overt spatial direction of $(p)$. However, Barberà identifies some semantic restrictions affecting this formal association that lead her to reframe the DR hypothesis proposed by r-loci accounts. According to her, variables with narrow scope in LSC - occurring in donkey sentences, quantified NPs, generic statements and kinds - do not formally establish (p) in the signing space. For instance, generic statements do not co-occur with index signs and, consequently, the entity is not localized in space (ex. 1). In fact, whenever the DR is localized, it is understood as referential (ex. 2):

WOMAN PLAY LIKE NOT

'Women do not like to play.'

(2) WOMAN IX3 PLAY LIKE NOT

'A/the/this/that woman does not like to play.' 
Importantly, the narrow/wide distinction predicts coreferential constraints arising from the position of the variable within a Discourse Representation Structure (DRS): variables with wide scope appear in the main DRS and are accessible for further coreference, while those with narrow scope appear in a subordinate DRS, and outside this scope, they are not available for coreferential relations. Since in LSC only DRs with wide scope establish (p), Barberà closes the chapter proposing a more accurate explanation for the semantic function of spatial locations: (p) is the overt manifestation of DRs attached to quantifiers with wide scope.

Chapter 5 analyzes the connection between spatial locations and the expression of definiteness. To this aim, a theoretical background for definiteness and deixis is presented. In DRT, both definite and indefinite NPs share the same semantic representation. For this reason, their difference needs to be explained by means of their conditions: a definite NP signals an existing DR (familiarity condition), whereas an indefinite NP signals novelty (unfamiliarity condition) of the DR. Moreover, they also differ in their descriptive content: in indefinites, it is asserted, while in definites, it is presupposed. The analysis proposed for the possible encoding of definiteness in LSC spatial locations is based on the notion of familiarity. The data provided by Barberà shows that the establishment of a DR in signing space does not denote a definite reading in LSC - (in)definiteness, however, can be expressed through a restricted set of determiners or specific nonmanual marking-. Unlike other SLs, LSC uses the lower frontal plane to establish both definites and indefinites (Figure 1); hence, there is no distinction between presupposition (for weak familiar DRs) and assertion (for strong familiar DRs) of existence concerning the spatial localization, since both possibilities establish (p). The upper frontal plane in LSC is, however, reserved for a subtype of indefinites. Regarding deixis, the author widely explores this notion and compares it to that of anaphora. The conclusion is that both kinds of references should not be distinguished, as both are related to definiteness and presuppose that the DR is familiar to the audience. The familiarity notion is attained in deixis by pointing at something which is prominent or salient; in the case of anaphora, by pointing at some previous linguistic mention. Therefore, they are just "different means of introducing entities into the discourse model". Concerning LSC, new DRs establishing (p) can appear without an overt antecedent. Weak familiarity antecedents can be inferred from the contextual environment and, later, accommodated into the model. In contrast to Liddell's (2003) proposal, Barberà provides sufficient data to prove that the interpretation of the pronominal index signs is done by the selection of a suitable DR (which they are linked to) among a set of context-available information and not by the directionality of a physical sign (that is deictically referring to the present object). This can show that all references are anaphoric to the discourse model (even deictic uses). 


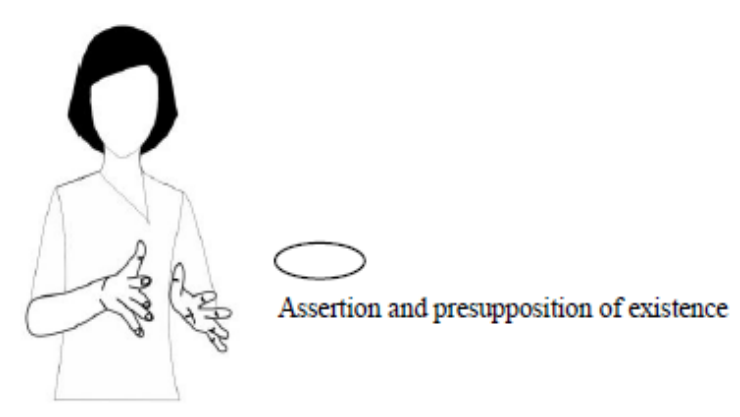

Figure 1. Definiteness marking in LSC signing space

By introducing specificity marking into the discussion, Chapter 6 proposes an additional adjustment of the formalization developed so far. As already indicated, under Barberà's analysis, the establishment of two opposed locations on the frontal plane is used in LSC to overtly denote (non-)specificity (Figure 2). This is further confirmed by comparing the morphophonological features directed to signing space. The analysis reveals the existence of two localization patterns: strong and weak, which directly correlate with the establishment of lower and upper locations. Therefore, strong localization is formally marked by the default location (p), whereas weak localization is marked by the feature (p)[up]. Building on DRT, which considers specificity as a scope-related issue, Barberà shows that weak and strong localizations mark not only scope distinctions, but also partitivity and identifiability. That is, the lower part of the frontal plane denotes identifiable and specific DRs (equated with wide scope), and it is also used in partitive constructions. The upper part, in turn, corresponds to non-specificity (narrow scope DRs), non-identifiability and non-partitivity.

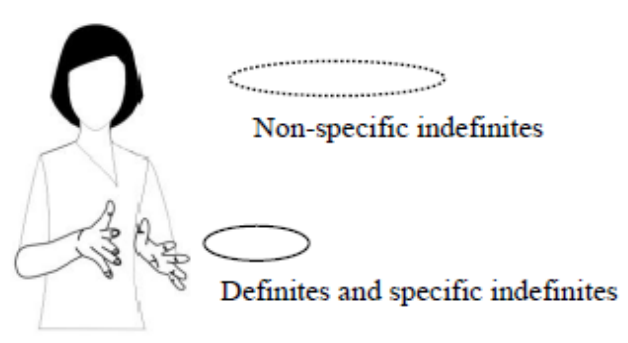

Figure 2. Definiteness and specificity marking on LSC frontal plane

Based on these findings, the author proposes a more detailed description of the functions undertaken by spatial locations. The DR hypothesis is then amended as follows: ( $p$ ) is the overt manifestation of wide scope denoting specificity, while (p)[up] is the overt manifestation of narrow scope denoting non-specific DRs.

Chapter 7 suggests two-fined grained hypotheses concerning (p) which help to offer a better analysis for LSC spatial locations - specifically, those on the horizontal plane-, since they take a discourse structure perspective. The objectives of the chapter are two-fold. First, discourse prominence takes lower spatial locations. Therefore, and contrary to what have been seen in previous 
chapters, the author shows that narrow scope variables attached to a prominent DR in discourse do not longer behave as narrow scope but rather as wide scope variables and, therefore, they are established in the [low] spatial location. Second, the nature of spatial locations is reconsidered when data from connected discourse is introduced into the picture. The conclusion is that the abstract point (p) does not correspond to an exact point in space and neither is it related to any specific direction of signs. Those locations which are associated to the most prominent DR in discourse, then, can be shifted and established in different points in space, and the result is completely irrelevant for (p): it is still related to a DR that can be found in the model, and yet it works independently from where we place it in the signing space.

Chapter 8 works as a summary of the main findings of the dissertation and adds interesting issues which have been raised along the study and that should be considered for future research.

As may be seen from the above, this book has overcome the challenge of uncovering the relation between the form of spatial locations in LSC and their semantic functions, having largely accomplished the objectives set out at the beginning. On the one hand, Barberà offers a systematic mapping of the morphophonological features directed to space and their particular semantic effects in the interpretation of LSC discourse. After an in-depth analysis, the results show that the establishment of spatial locations directly corresponds to the semantic notion of scope, and that the spatial morpheme (p) is responsible for the linguistic marking of both specificity and topicality. Barberà's findings convincingly prove that spatial locations are incorporated into the grammar, offering new and well-grounded arguments in favor of r-loci accounts.

Additionally, the formalization presented, increasingly refined as the book develops, represents an important step forward to bridge a gap that has existed in previous analyses of space in SLs. As a result, it opens the door to crosslinguistically compare the behavior of spatial locations in other SLs. Importantly, the book also reveals the importance of adding the analysis of visual languages to the global picture of natural language semantics. Given that referential indices are overtly expressed in sign languages, something that does not hold for their spoken counterparts, investigation into them can elucidate, and even transform, our understanding of the semantic underpinnings of (co-)reference. In conclusion, we consider this book as an essential reference to readers interested both in formal approaches to meaning in discourse and the nature and function of space in sign language.

\section{References}

Kamp, Hans \& Uwe Reyle. 1993. From discourse to logic. Introduction to modeltheoretic semantics of natural language, formal logic and discourse representation theory. Dordrecht: Kluwer Academic Press. https://doi.org/10.1007/978-94-011-2066-1 
Liddell, Scott. 1990. "Four Functions of a Locus: Reexamining the Structure of Space in ASL". In C. Lucas (ed.), Sign Language Research: Theoretical Issues, 176-198. Washington D.C.: Gallaudet University Press.

Liddell, Scott. 2003. Grammar, Gesture and Meaning in American Sign Language. Cambridge: Cambridge University Press.

https://doi.org/10.1017/cbo9780511615054.012

Lillo-Martin, Diane \& Edward Klima. 1990. "Pointing Out Differences: ASL Pronouns in Syntactic Theory". In S. Fischer \& P. Siple, Theoretical Issues in Sign Language Research, Vol. 1: Linguistics, 191-210. Chicago: University Chicago Press. 